Research Paper

\title{
Flotillin-2 Expression in the Human Gut: from a Cell Model to Human Tissue in Health and Inflammatory Bowel Diseases
}

\author{
Annika Gauss ${ }^{1}$, Inga Buchholz ${ }^{1}$, Alexandra Zahn¹, Gerd Schmitz², Wolfgang Stremmel1, Joachim \\ Fuellekrug${ }^{1}$, Robert Ehehalt ${ }^{\boxplus}$ \\ 1. University Hospital Heidelberg, Department of Gastroenterology, Im Neuenheimer Feld 410, 69120 Heidelberg, Germany; \\ 2. University Hospital Regensburg, Department of Clinical Chemistry and Laboratory Medicine, Franz-Josef-Strauss-Allee 11, 93053 Re- \\ gensburg, Germany.
}

$\square$ Corresponding author: Robert Ehehalt, M.D., University Hospital Heidelberg, INF 410, 69120 Heidelberg, Germany; E-mail: Robert.ehehalt@med.uni-heidelberg.de.

(c) Ivyspring International Publisher. This is an open-access article distributed under the terms of the Creative Commons License (http://creativecommons.org/ licenses/by-nc-nd/3.0/). Reproduction is permitted for personal, noncommercial use, provided that the article is in whole, unmodified, and properly cited.

Received: 2013.03.29; Accepted: 2013.07.22; Published: 2013.08.03

\begin{abstract}
Background and aims: The etiopathogenesis of inflammatory bowel diseases (IBD) remains largely unexplained. Flotillins (flotillin-I and flotillin-2) are ubiquitous proteins which have been linked to inflammation and regeneration. We hypothesized that alterations in the expression of flotillin-2 in enterocytes may be related to the pathogenesis of IBD as a classical example of an inflammatory disorder of mostly unknown origin.

Methods: Cell and tissue localization of flotillin-2 (and -1 ) were investigated by immunofluorescent staining in I. polarized and unpolarized $\mathrm{CaCo}-2 \mathrm{w}$ cells as a model of human enterocytes (native and after TNFa stimulation) and 2. intestinal biopsies from controls, patients with ulcerative colitis (UC) and patients with Crohn's disease (CD). For quantification of flotillin-2, we analyzed its expression in ileal and colonic biopsies from controls, UC patients and CD patients using real-time RT-PCR, Western blot and indirect immunofluorescence.

Results: In polarized $\mathrm{CaCo}-2 \mathrm{w}$ cells and human enterocytes in biopsies, flotillins were localized at the basolateral membrane and on subapical vesicles, but not in the apical membrane. Flotillin-2 expression did not differ between UC patients, CD patients and controls. However, it was significantly higher in colonic biopsies compared to ileal biopsies in all groups.

Conclusions: By virtue of its abundant expression in enterocytes, flotillin- 2 must have an essential function in intestinal physiology, especially in the colon. Yet our data could not link flotillin-2 to the pathogenesis of IBD.
\end{abstract}

Key words: Inflammatory bowel disease (IBD); Ulcerative colitis; Crohn's disease; Lipid raft; Flotillin-1; Flotillin-2.

\section{Introduction}

Flotillin-1 (reggie-2) and flotillin-2 (reggie-1) are ubiquitous, highly conserved proteins that were first described to be upregulated in regenerating retinal ganglion cells of a goldfish. ${ }^{[1-3]}$ Both flotillins were shown to be associated with lipid rafts and have been used as raft marker proteins. ${ }^{[4]}$ Flotillins are associated with the cytosolic side of the plasma membrane, ${ }^{[5]}$ but are also present intracellularly in different vesicular compartments like endosomes, lysosomes and phagolysosomes. ${ }^{[6-12]}$ They appear to be expressed in all mammalian cells and tissues and possess a wide spectrum of functions in cell signaling, endocytosis, 
phagocytosis and interactions with the cytoskeleton. ${ }^{[5,13]}$ However, there remain many open questions and controversies about flotillin function.

In mammals, flotillin-2 is widely expressed in numerous different tissues, while flotillin- 1 appears to be more restricted.[14] Flotillin-1 is highly expressed in brain, heart, placenta and hematopoietic cells. ${ }^{[15,16]}$ In contrast, PCR analysis of flotillin-2 in murine and human tissues showed ubiquitous expression.[17]

To date, several diseases have been described in which the expression of flotillins was distinctly altered. Among them are malignant tumors such as breast cancer, where flotillin-1 was found to be overexpressed in $61 \%$ of the samples examined. [18] Flotillin-1 has also been demonstrated to be overexpressed in spinal cord cells of Lewis rats with experimentally induced autoimmune encephalitis as an example of inflammation. ${ }^{[19]}$ In a recently published manuscript, Song et al. showed that flotillin-1 was upregulated in esophageal squamous cell carcinoma (ESCC). It was demonstrated to activate TNFa receptor signaling and to sustain activation of NF-kB in ESCC cells. [20]

The disease group of IBD comprises ulcerative colitis (UC) and Crohn's disease (CD). Both are chronic, non-infectious inflammatory diseases of the human intestine. Their etiopathogenesis still remains largely unknown. However, it is becoming clearer that a combination of an intestinal barrier dysfunction with an over-activated immune system might play a key role.[21]

Why examine flotillins in IBD? Data are very scarce on flotillins in IBD so far; however, a few data are available on a potential role of lipid rafts in IBD. ${ }^{[22,23]}$ Lipid rafts are abundant in enterocytes, especially in their apical cell membranes. ${ }^{[24]}$ Representing signal transduction platforms, lipid rafts are fundamental players in processes of inflammation and immunity.[25] The effects of many mediators of inflammation which are considered to have roles in the pathogenesis of IBD - like interleukin-1 $\beta, \mathrm{TNFa}$, TRL2, CD14 und TLR4 - seem to depend on rafts.[26-28] Lipid rafts may also induce signal transduction processes that are necessary for the intrusion of pathogens into their target cells. ${ }^{[24,29,30]}$ Pathological changes to lipid raft function might thus interfere with epithelial barrier integrity against intestinal bacteria. A link between alterations in lipid raft function in the intestinal tract and IBD may also be suggested due to the known therapeutic effects of phosphatidylcholine (PC), the latter seeming to interfere with raft composition and function and thus to alleviate inflammation. [31-33]

Unpublished microarray data obtained via Affymetrix U133 gene chip expression analysis of RNA samples from human intestinal biopsies suggest that flotillin-2 may have a role in IBD (method described in ${ }^{[34,35])}$. They showed reduced flotillin-2 expression compared to controls in a limited number of biopsies taken from non-affected mucosal areas in patients suffering from UC. Even though flotillin-2 gene expression was predominantly low in the colon, it was also lower in ileum specimens from patients with ulcerative colitis compared to ileum specimens from controls.

Flotillin-2 as a player in regeneration, differentiation and inflammation, which are all essentially involved in IBD, thus appeared to be an interesting target for IBD research with the following primary objectives: 1 . To localize the protein in vitro in native and TNFa-treated CaCo-2w cells, representing a model of human intestinal epithelial cells with or without inflammation; 2 . To compare the results to in vivo localization data in human intestinal biopsies from controls and patients with UC or CD. 3. To analyze flotillin-2 expression in biopsies from healthy subjects and IBD patients at the RNA and, especially, protein level.

\section{Materials and Methods}

\section{Cell culture}

Caco- $2 \mathrm{w}$ cells are a subclone of human Caco-2BBe cells with a well-differentiated phenotype (kindly provided by J. R. Turner, University of Chicago, IL). [33] The cells were maintained under standard tissue culture conditions using Dulbecco's Modified Eagle's Medium (DMEM) supplemented with 4.5 $\mathrm{g} / \mathrm{L}$ glucose, $10 \%$ heat-inactivated fetal calf serum, 2 $\mathrm{mM}$ glutamin, $0.1 \mathrm{mM}$ non-essential amino acids, 1 $\mathrm{mM}$ sodium pyruvate and antibiotics $(100 \mathrm{IU} / \mathrm{ml}$ streptomycin and $100 \mu \mathrm{g} / \mathrm{ml}$ penicillin). The cells were cultivated in plastic flasks at $37{ }^{\circ} \mathrm{C}$ under $5 \%$ $\mathrm{CO}_{2}$.

For polarization on semipermeable filters, Caco-2w cells were seeded in 6-well Vitaris transwell polyester membrane inserts (pore size $0.4 \mu \mathrm{m}$; Vitaris AG, Baar, Switzerland) and cultured for 2 to 3 weeks. TNFa (Promega, Madison, WI) was applied at 1 $\mathrm{ng} / \mathrm{ml}$ or $2 \mathrm{ng} / \mathrm{ml}\left(1 \mathrm{~h}, 37^{\circ} \mathrm{C}\right)$ on subconfluent Caco- $2 \mathrm{w}$ cells.

\section{Human intestinal biopsies}

All ileal and colonic biopsies were acquired endoscopically from patients visiting the Department of Endoscopy of the University Hospital Heidelberg. They were taken during colonoscopy and immediately transferred into liquid nitrogen, either as is or in RNAlater ${ }^{\mathrm{TM}}$ for RT-PCR (Ambion, Frankfurt, Germany). Prior to examination they were stored at -80 ${ }^{\circ} \mathrm{C}$. Since most proteins are differentially expressed 
within the colon, colon samples were taken from the transverse colon in all patients. In order to avoid gene expression influenced by inflammatory processes, biopsies were taken from non-inflamed mucosal areas or areas with as little macroscopic inflammation as possible.

Three groups of patients were examined: healthy controls, patients with UC, and patients with CD. Diagnoses of UC and CD were made on the basis of patient histories, endoscopic and histologic findings. These patients had different disease activities when they were examined. Controls were patients admitted for colonoscopy screening or undergoing colonoscopy for various abdominal complaints. None of the controls had a history of IBD, and in none of the controls did we find evidence of inflammation. Demographic and clinical characteristics of the subjects whose specimens were used for immunoblotting are shown in Table 1. As it would not add significant information, corresponding detailed clinical data of patients whose samples were used for PCR are not shown.
For Western blot analysis, we included altogether 32 ileal (11 controls, 11 CD patients and $10 \mathrm{UC}$ patients) and 30 colonic biopsies (10 controls, $10 \mathrm{CD}$ patients and $10 \mathrm{UC}$ patients). RT-PCR analyses were performed from 72 biopsies (12 subjects in each of the three groups from the ileum and colon, respectively). The study was performed in accordance with the Declaration of Helsinki and approved by the local Ethics Committee.

\section{Antibodies}

Table 2 summarizes all the antibodies used in this study with their application, sources and dilutions. Antibodies against flotillin-1 were raised in rabbits according to standard procedures using a synthetic peptide (Sigma-Genosys, Cambridge, UK) coupled to keyhole limpet hemocyanin. The peptide corresponded to the C-terminus of human flotillin-1 and contained an additional N-terminal cysteine (CVNHNKPLRTA). Affinity purification of the antiserum was as recommended by the manufacturer on a peptide-agarose column (Pierce, Rockford, IL).

Table I. Characteristics of the patients whose biopsies were used for Western blot analysis. UC: ulcerative colitis; CD: Crohn's disease; f: female, m: male; y: yes, n: no; d.n.a.: does not apply. The term "active disease" was defined on the basis of colonoscopic and histologic findings.

\begin{tabular}{lllllll}
\hline & ileum & & \multicolumn{3}{l}{ colon } \\
\hline & controls & UC & $C D$ & controls & UC & CD \\
\hline $\mathrm{n}$ & 11 & 10 & 11 & 10 & 10 & 10 \\
$\operatorname{sex}(\mathrm{f} / \mathrm{m})$ & $4 / 7$ & $5 / 5$ & $8 / 3$ & $6 / 4$ & $5 / 5$ & $5 / 5$ \\
age (years) & $42.7 \pm 3.3$ & $39.9 \pm 4.0$ & $38.6 \pm 4.2$ & $41.8 \pm 3.0$ & $38.6 \pm 5.1$ & $40.9 \pm 4.1$ \\
(neo-)terminal ileal manifestation (y/n) & d.n.a. & $0 / 10$ & $7 / 4$ & d.n.a. & $1 / 9$ & $9 / 1$ \\
colonic manifestation (y/n) & d.n.a. & $10 / 0$ & $9 / 2$ & d.n.a. & $10 / 0$ & $7 / 3$ \\
active disease $(\mathrm{y} / \mathrm{n})$ & d.n.a. & $8 / 2$ & $9 / 2$ & d.n.a. & $9 / 1$ & $10 / 0$ \\
\hline
\end{tabular}

Table 2. Sources and specifications of antibodies used in the study.

\begin{tabular}{|c|c|c|c|c|c|}
\hline & reactivity & origin & usage & source & dilution \\
\hline \multicolumn{6}{|l|}{ Primary Antibodies } \\
\hline CEA, A0115 & $\mathrm{h}$ & $\mathrm{rb}$ & IF & DAKO, Glostrup, Denmark & $1: 400$ \\
\hline Flotillin-1 & $\mathrm{h}$ & $\mathrm{rb}$ & IF & provided by Kai Simons, Dresden, Germany & 1:1000 \\
\hline Flotillin-2, 610383 & $\mathrm{~h}$ & $\mathrm{~m}$ & IF, WB & BD Biosciences Pharmingen, Heidelberg, Germany & $\begin{array}{l}\text { IF: } 1: 100 \\
\text { WB: } 1: 5000\end{array}$ \\
\hline Na-K-ATPase, MA3-928 & $\mathrm{h}$ & $\mathrm{m}$ & IF & ABR Affinity BioReagents, Golden, USA & $1: 50$ \\
\hline B-actin, A55441 & $\mathrm{h}$ & $\mathrm{m}$ & IF, WB & Sigma-Aldrich, St Louis, USA & $1: 100,000$ \\
\hline \multicolumn{6}{|l|}{ Secondary antibodies } \\
\hline cy2 a-mouse, 715-225-151 & $\mathrm{m}$ & $\mathrm{d}$ & IF & Jackson ImmunoResearch, West Grove, USA & $1: 230$ \\
\hline cy2 a-rabbit, 711-225-152 & $\mathrm{rb}$ & d & IF & Jackson ImmunoResearch, West Grove, USA & $1: 250$ \\
\hline cy3 a-mouse, 715-165-151 & $\mathrm{m}$ & d & IF & Jackson ImmunoResearch, West Grove, USA & 1:1000 \\
\hline cy3 a-rabbit, 711-165-152 & $\mathrm{rb}$ & $\mathrm{d}$ & IF & Jackson ImmunoResearch, West Grove, USA & 1:1000 \\
\hline anti-mouse HRP, 115-035-003 & $\mathrm{m}$ & g & WB & Jackson ImmunoResearch, West Grove, USA & $1: 5000$ \\
\hline
\end{tabular}

h - human; m - mouse; rb - rabbit; d - donkey; g - goat; IF - immunofluorescence; WB - Western blot. 


\section{Indirect immunofluorescence}

Immunofluorescence was used to localize flotillin-2 and flotillin-1 in cultured cells and human intestinal biopsies. For confocal microscopy, CEA and Na-K-ATPase were used as apical and basolateral membrane markers, respectively.

Cells grown on coverslips were washed twice with PBS and then fixed and permeabilized with ice-cold methanol for $2 \mathrm{~min}$ in $-20{ }^{\circ} \mathrm{C}$. Unspecific binding was blocked for $30 \mathrm{~min}$ at room temperature with PBS containing $0.01 \%$ saponin, $0.2 \%$ gelatin and $5 \mathrm{mg} / \mathrm{ml} \mathrm{BSA}$. Primary antibodies (see Table 2) in SGB solution were added for $1 \mathrm{~h}$ at room temperature. After primary antibody binding, the cells were washed 3 times with PBS containing 0.01\% saponin and $0.2 \%$ gelatin.

The fluorescent-conjugated secondary antibodies (Cy ${ }^{\mathrm{TM}} 3$ anti-mouse) were added for $1 \mathrm{~h}$ at room temperature. Subsequently, the cells were washed twice with SG solution, twice with PBS and once with water, and mounted on microscopic slides using Mowiol (Calbiochem, Bad Soden, Germany). The polarized Caco- $2 \mathrm{w}$ cells on the filters were prepared as described above. For immunohistochemistry, frozen biopsies were embedded in Tissue Tek (Sakura Finetek, Zoeterwoude, NL) on dry ice. Five micron cryosections were prepared at $22{ }^{\circ} \mathrm{C}$ in a cryostat (model CM 3050S, Leica, Bensheim, Germany) and mounted on glass slides. Fixation and permeabilization were performed using acetone for $5 \mathrm{~min}$ and then methanol for $2 \mathrm{~min}$, both at $-20^{\circ} \mathrm{C}$. Slides were then blocked with $4 \%$ pig normal serum (DAKO, Glostrup, Denmark) in SGB solution for $30 \mathrm{~min}$. Primary and secondary antibodies were applied as indicated above. In some experiments, Phalloidin Fluo Probes 547 (KMF Laborchemie, Lohmar, Germany) was added to the secondary antibody solution in a 1:10,000 dilution. Nuclei were stained with $1 \mu \mathrm{g} / \mathrm{ml}$ Hoechst 33342 (Roche, Mannheim, Germany).

All images of cultured cells and tissues except those from confocal microscopy were acquired on an Olympus IX50 fluorescence microscope using a $60 \mathrm{x}$ oil immersion objective and analySIS software from Soft Imaging System (Muenster, Germany). Confocal microscopic images were obtained on a Leica TCS SP2 confocal microscope (Leica Microsystems, Wetzlar, Germany) using the HCX PL APO $63 \times 1.32$ oil objective. Images were arranged with Adobe Photoshop (Adobe Systems, Mountain View, CA).

\section{Immunoblotting}

For immunoblotting, proteins were extracted from intestinal biopsies. Each biopsy was transferred into a tube with $200 \mu \mathrm{l}$ of sample buffer $(125 \mathrm{mM}$
Tris- $\mathrm{HCl} \mathrm{pH}$ 6.8, 4\% SDS, 20\% glycerin), vortexed thoroughly and incubated at $95{ }^{\circ} \mathrm{C}$ for $5 \mathrm{~min}$. After centrifugation, the supernatants were transferred to new tubes for protein determination using bicinchoninic acid (BCA).[36] Equal amounts of protein $(5 \mu \mathrm{g})$ were used from each subject. In order to optimize the comparability between patient groups and between different gels, the 12 lanes of each gel were loaded as follows: 3 to 4 control samples, 3 to 4 UC samples, 3 to 4 CD samples, 1 defined total Caco- $2 \mathrm{w}$ cell lysate sample as well as a weight marker. The proteins were then separated by sodium dodecyl sulfate-polyacrylamide gel electrophoresis (SDS-PAGE) in $10 \%$ gels and transferred to nitrocellulose membranes. After transfer, the nitrocellulose sheets were stained with Ponceau S (Serva, Heidelberg, Germany) to visualize the protein bands. The membranes were subsequently incubated with anti-flotillin-2 (1:5000 in $2 \%$ skim milk) and anti-ß3-actin (1:100,000 in $2 \%$ skim milk) overnight at $4{ }^{\circ} \mathrm{C}$, followed by a $1 \mathrm{~h}$ incubation period with horseradish peroxidase-conjugated secondary antibody (1:5000 in $2 \%$ skim milk) at room temperature. After chemiluminescent detection with a commercial ECL reagent following the manufacturer's instructions (Amersham Biosciences, Buckinghamshire, UK), the films were scanned and bands were quantified by determination of density using MCID ${ }^{\mathrm{TM}}$ Analysis Evaluation 7.0. For internal control, densitometry values obtained for flotillin- 2 in every specimen were related to the value obtained for $B$-actin from the same patient. For comparison, these values were related to the flotillin-2/ß-actin values in the total cell lysates.

\section{Quantitative real-time RT-PCR}

For quantification of human flotillin-2 mRNA transcripts, total RNA was isolated from intestinal biopsies using the RNAqueous RNA isolation kit, and subsequently purified with the DNA-free kit (both from Ambion, Cambridgeshire, UK) according to the instructions of the manufacturer. Total RNA concentrations were determined photometrically at $260 \mathrm{~nm}$. For each biopsy, a volume equivalent to $1 \mu \mathrm{g}$ of total RNA was used for reverse transcription using the Transkriptor First Strand cDNA Synthesis Kit (Roche Diagnostics, Mannheim, Germany). Calibration plasmids containing the sequence of the human flotillin-2 gene were prepared as follows: DNA was isolated from CaCo- 2 w cells using the QIAamp DNA mini Kit (Qiagen, Hilden, Germany). The desired sequence was amplified by PCR using the Omniscript-RT Kit (Qiagen, Hilden, Germany). The amplified DNA fragment was purified by agarose gel electrophoresis. It was then inserted into the TA vector using the pGEMT vector ligation system (Promega, Mannheim, Germany). 
Vectors were subsequently transformed into E. coli, and plasmids were isolated from E. coli cultures with the NucleoSpin Plasmid Kit (Macherey-Nagel, Düren, Germany).

Real-time PCR was performed using the LightCycler 2.0 system (Roche, Mannheim, Germany). Transcripts were detected with SYBR Green I and normalized to human $\beta$-actin. Flotillin-2/ß-actin ratios were calculated using Relative Quantification Software Version 1.0 und GIMP 2.2.

Primer sequences $\left(5^{\prime}\right.$ to $\left.3^{\prime}\right)$ : human flotillin-2 ACAGCTGAGGCCCAGTTG, TGAGCTCCTTGTCC GTACG; human B-actin AGGATGCAGAAGGAGAT CACTG, GGGTGTAACGCAACTAAGTCATAG.

\section{Documentation and statistics}

Documentation, data processing and graphic presentation of results were performed using Apache OpenOffice Calc., SPSS version 15 (SPSS Inc., Chicago, IL) and GraphPad Prism 3.0 (GraphPad Inc., San Diego, CA). Data are presented as means and standard errors of the mean (SEM). Non-parametric Kruskal-Wallis with Dunn's post-test was used for comparisons between the 3 patient groups and the Mann-Whitney-U test for comparison of ileal vs. colonic biopsies. The level of significance was set at $p \leq$ 0.05 .

\section{Results}

\section{Localization of flotillin-2 in unpolarized $\mathrm{Ca}$ - Co-2w cells}

Immunostaining revealed distinct flotillin-2 expression in the plasma membrane as well as in intracellular vesicular structures in unpolarized $\mathrm{CaCo}-2 \mathrm{w}$ cells (Figure 1A). The experiments on unpolarized $\mathrm{CaCo}-2 \mathrm{w}$ also showed that the localization of flotillin-2 was cell-cell contact dependent. Figure 1B

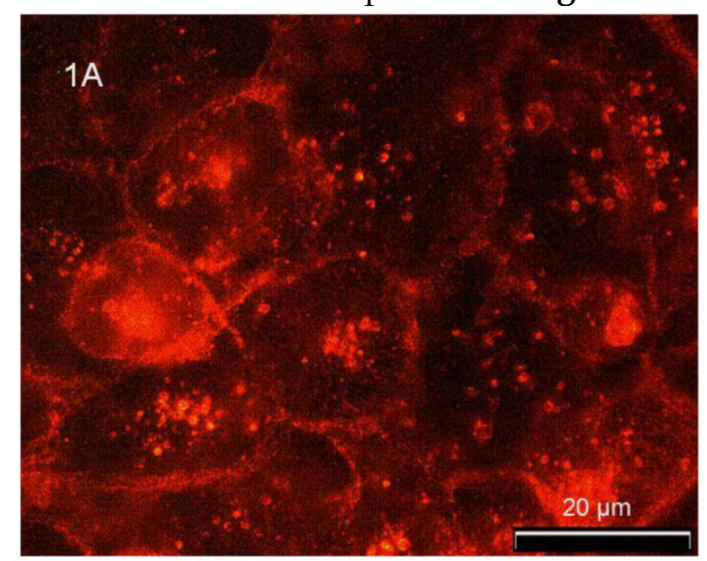

demonstrates how cell-cell contacts caused increased expression of flotillin-2 in the area of the cell adjoining a neighboring cell. In parallel, we observed a reduction in flotillin-2 expression within intracellular vesicles.

\section{Cell differentiation influences flotillin-2 locali- zation in CaCo-2w cells}

In order to analyze the effects of cell differentiation on flotillin-2 localization, polarized $\mathrm{CaCo}-2 \mathrm{w}$ cell monolayers were grown on filters and examined by immunofluorescence. To allocate flotillin-2 to different cell membrane domains (apical versus basolateral), our intent was to co-localize flotillin-2 with $\mathrm{Na}-\mathrm{K}-\mathrm{ATPase}$ as a basolateral marker. As both available antibodies were monoclonal mouse antibodies, using the same secondary antibody for fluorescence detection would have made it impossible to discern between flotillin-2 and Na-K-ATPase. Therefore a flotillin-1 antibody (rabbit) was used for the basolateral co-localization studies. In unpolarized $\mathrm{CaCo}-2 \mathrm{w}$ cells and in a sample colonic biopsy, flotillin-1 and flotillin-2 co-localized almost completely (see Figure 2). Based on this close relation between flotillin-1 and flotillin-2, co-localization was also assumed in polarized $\mathrm{CaCo}-2 \mathrm{w}$ cells, so basolateral co-localization experiments were performed with flotillin-1 and Na-K-ATPase antibodies. Figure 3A displays co-localization of flotillin-1 and Na-K-ATPase in basolateral cell membranes of filter-grown polarized CaCo-2w cells. Flotillin-1 (and, correspondingly, flotillin-2) could not be identified within the apical plasma membrane and only barely within the basal plasma compartment, but were more evident within vesicle-like structures in the subapical cytoplasm (view Figure 3B).

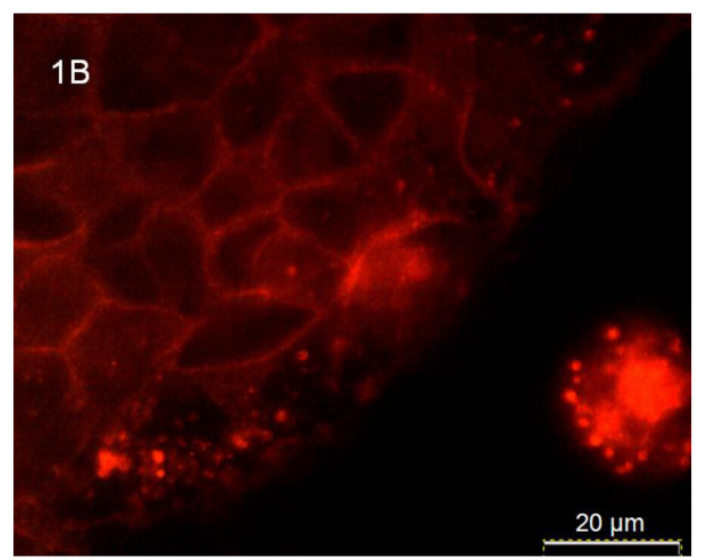

Fig I. (A) Immunofluorescence demonstrating the distribution of flotillin-2 in unpolarized CaCo-2w cells. Note that flotillin-2 is found in association with the plasma membrane and in intracellular vesicles. (B) shows that flotillin-2 localization in unpolarized $\mathrm{CaCo}-2 \mathrm{w}$ cells depends on cell-cell interactions. The $\mathrm{CaCo}-2 \mathrm{w}$ cells in the upper left of the image are in direct contact with neighboring cells. Accordingly, flotillin- 2 is concentrated in their plasma membranes. The row of cells closest to the edge contains most of the flotillin- 2 in cytoplasmic vesicles and only a small amount in the plasma membranes in close proximity to the neighboring cells on the left side. In the isolated cell in the bottom right of the image, flotillin-2 seems to be expressed only in cytoplasmic vesicles, but not in the plasma membrane. 

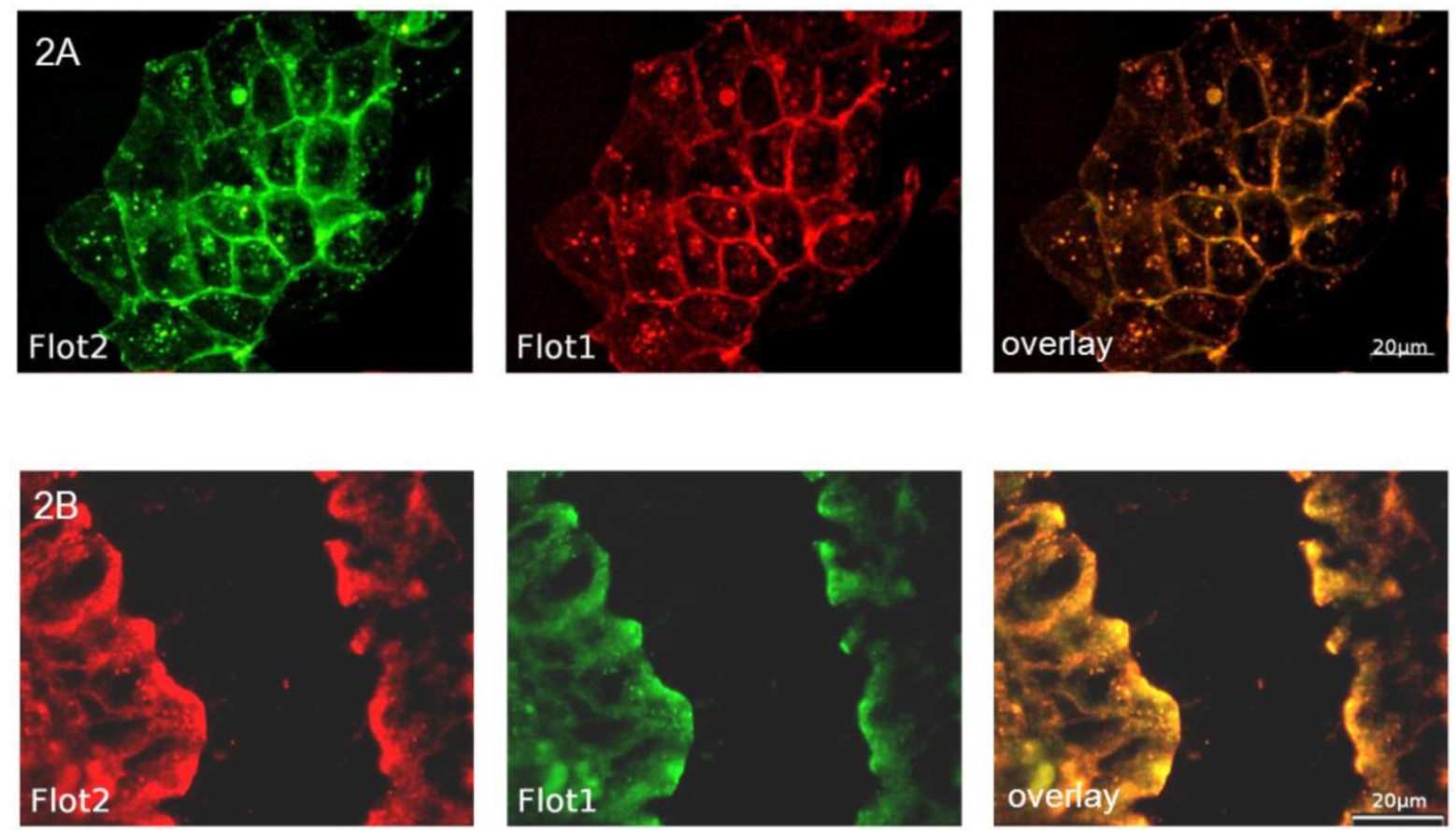

Fig 2. (A) shows the co-localization (overlay yellow, on the right) of flotillin-2 (green) and flotillin-I (red) in unpolarized CaCo-2w cells. (B) demonstrates the co-localization (overlay yellow, on the right) of flotillin-2 (red) and flotillin-I (green) in a section of a human colonic biopsy.
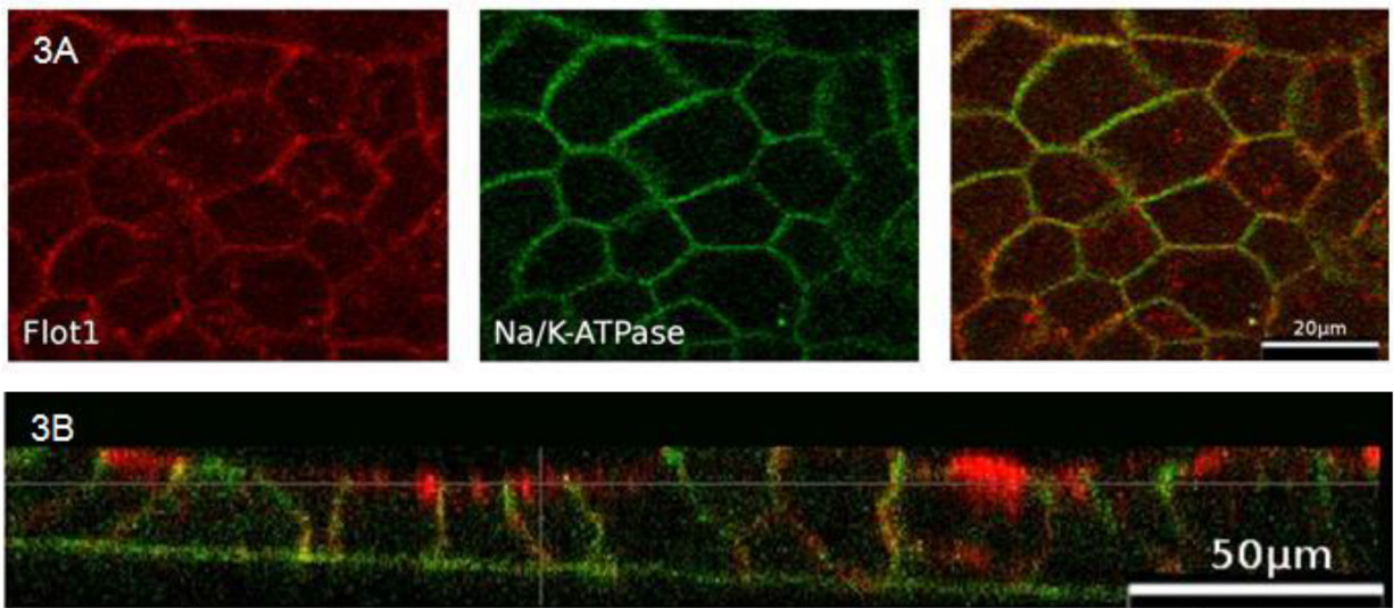

Fig 3. (A) Polarized CaCo-2w cells with localization of flotillin-I (red), Na-K-ATPase (green) and flotillin-I and Na-K-ATPase immunofluorescence overlay (right). Flotillin-I and Na-K-ATPase co-localize in the basolateral membrane. (B) Confocal microscopy image of polarized CaCo-2w cells. Flotillin-I (red) is localized in a subapical compartment, but not in the apical membrane. Na-K- ATPase (green) marks the basolateral membrane.

\section{Flotillin-2 in a cell model of intestinal inflam- mation}

As the major aim of the present study was to evaluate a potential role of flotillin-2 in the pathogenesis of IBD, an in vitro model of intestinal inflammation was used based on the hypothesis that expression and localization of flotillin-2 may be influenced by inflammation. However, treatment of CaCo- $2 \mathrm{w}$ cells with TNFa for $1 \mathrm{~h}$ prior to fixation for immunofluorescence did not result in an altered localization of flotillin-2 within the cells (Figure 4). Based on this lack of change, the hypothesis was not pursued further in the present study.

\section{Localization of flotillin-2 in human enterocytes from biopsies}

Immunohistochemical analysis of human enterocytes in endoscopic biopsies from the ileum and colon revealed - as in the in vitro experiments - two reservoirs of flotillin-2: the cell membrane and intracellular vesicles. This is shown in exemplary biopsy cryosections, in which flotillin-2 localization was visualized in relation to enterocyte nuclei stained with Hoechst and the apical membrane markers CEA and actin (Figures 5A-D). Using confocal microscopy analysis and co-localization studies of flotillin-1 and the basolateral membrane marker Na-K-ATPase, we 
also found significant flotillin-1 enrichment in basolateral enterocyte membranes (Figure 6). As elabo- rated above, we suggest that these results could be translated to the localization of flotillin-2.
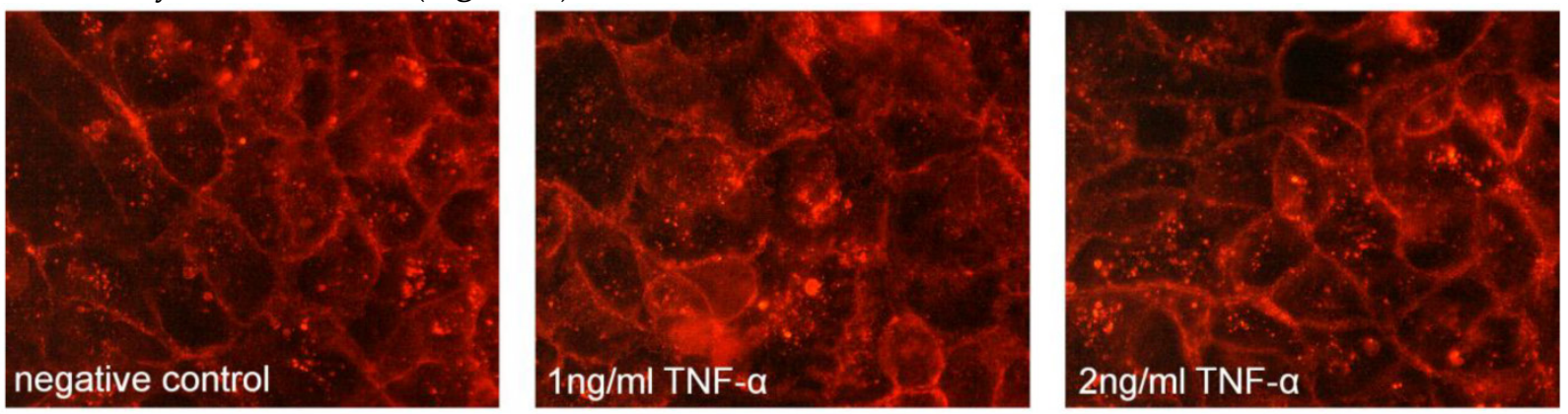

Fig 4. No shift in flotillin-2 expression (red) was noted in unpolarized CaCo-2w cells pretreated with TNFa at I ng/ml and $2 \mathrm{ng} / \mathrm{ml}$ for I h prior to fixation for immunostaining when compared to untreated cells (negative control).
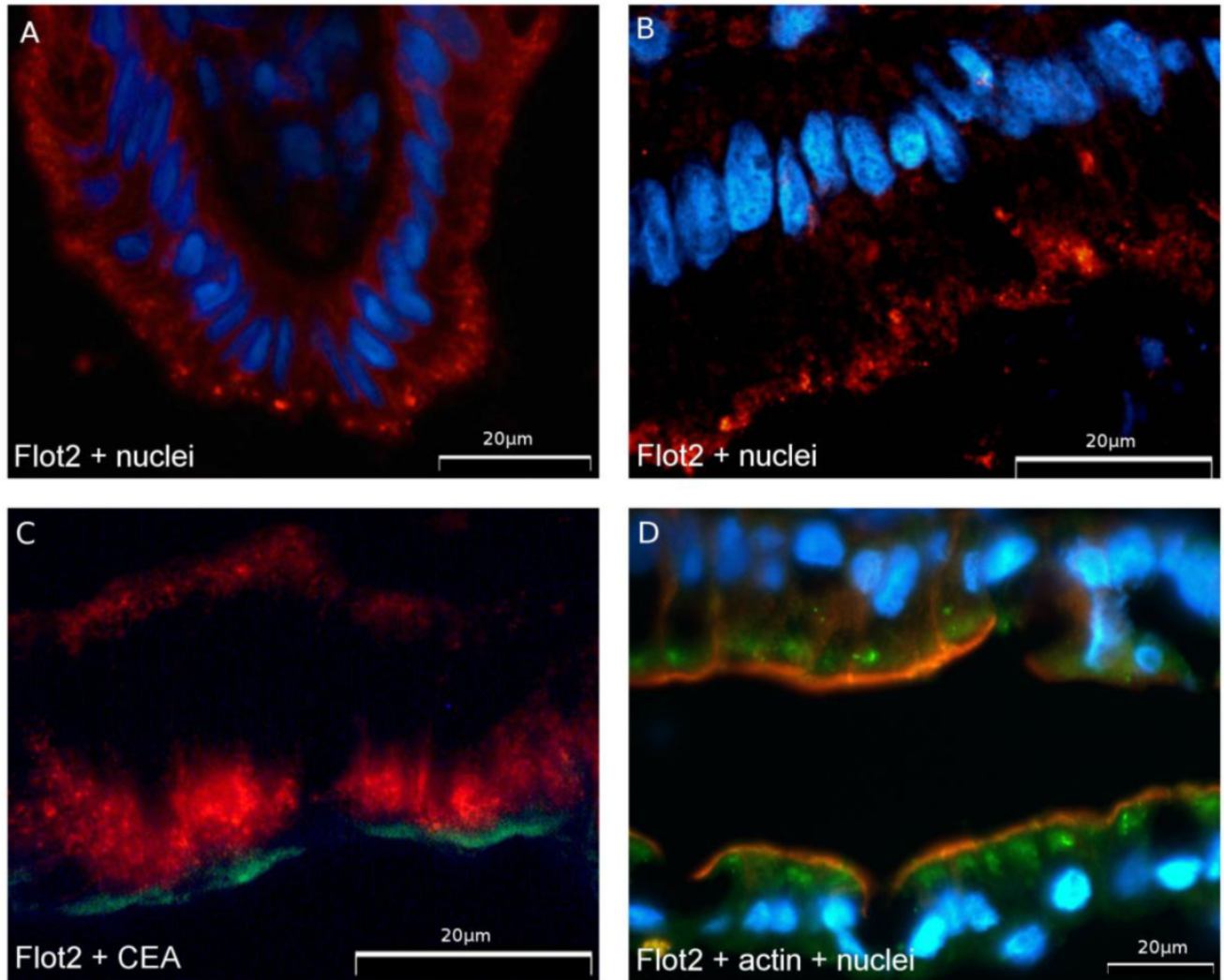

Fig 5. Flotillin-2 is localized in subapical vesicles and in the basolateral membrane in human enterocytes. (A and B) Expression of flotillin-2 (red) in colonocytes in a human biopsy. Blue nuclear counterstaining was performed with Hoechst. Flotillin-2 accumulates in subapical vesicles. (C) shows flotillin-2 (red) in relation to CEA as an apical cell membrane marker (green). Note that there is no overlay. (D) shows flotillin-2 in green, actin stained by phalloidin in light red as an apical cell membrane marker and blue Hoechst counterstaining of nuclei.
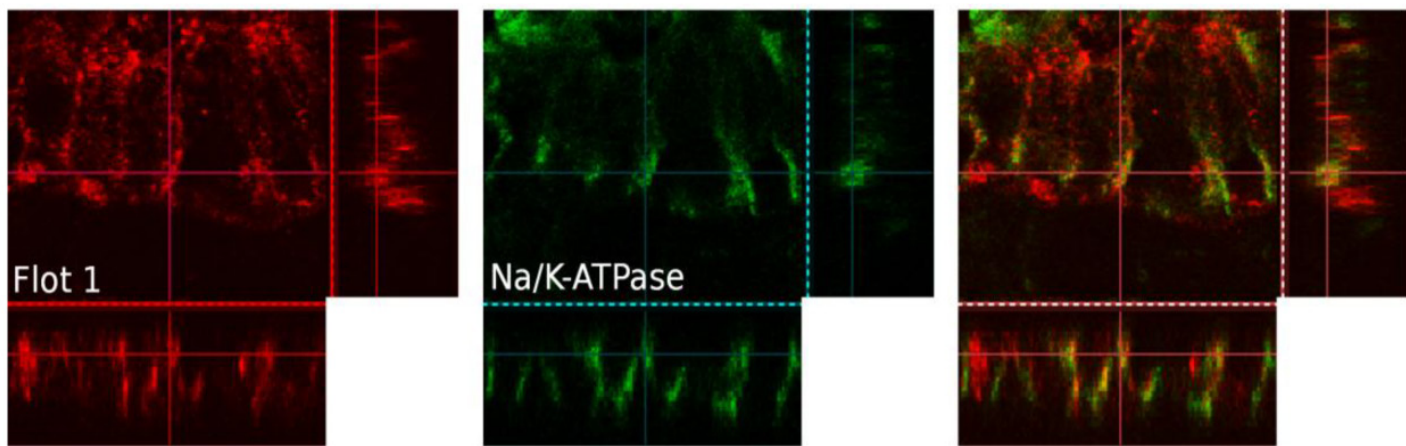

Fig 6. Three-dimensional, confocal-microscopic images of a human colonic epithelial layer in a human endoscopic biopsy. Flotillin-I is marked in red, Na-K-ATPase in green, immunofluorescence overlay on the right. Na-K-ATPase was used a marker of the basolateral cell membrane. 


\section{Localization of flotillin-2 in enterocytes in IBD patients compared to controls}

Three colonic biopsies and three ileal biopsies ( 3 controls, 3 UC patients and 3 CD patients) were analyzed by immunohistochemistry and compared to each another. For technical reasons, this evaluation focused on the subapical localization of flotillin-2. However, no difference was evident in the distribution pattern of flotillin-2 expression between IBD patients and the control subjects (images not shown).

\section{Expression of flotillin-2 at the RNA level - comparison between IBD patients and con- trols}

Human intestinal tissue specimens were evalu-
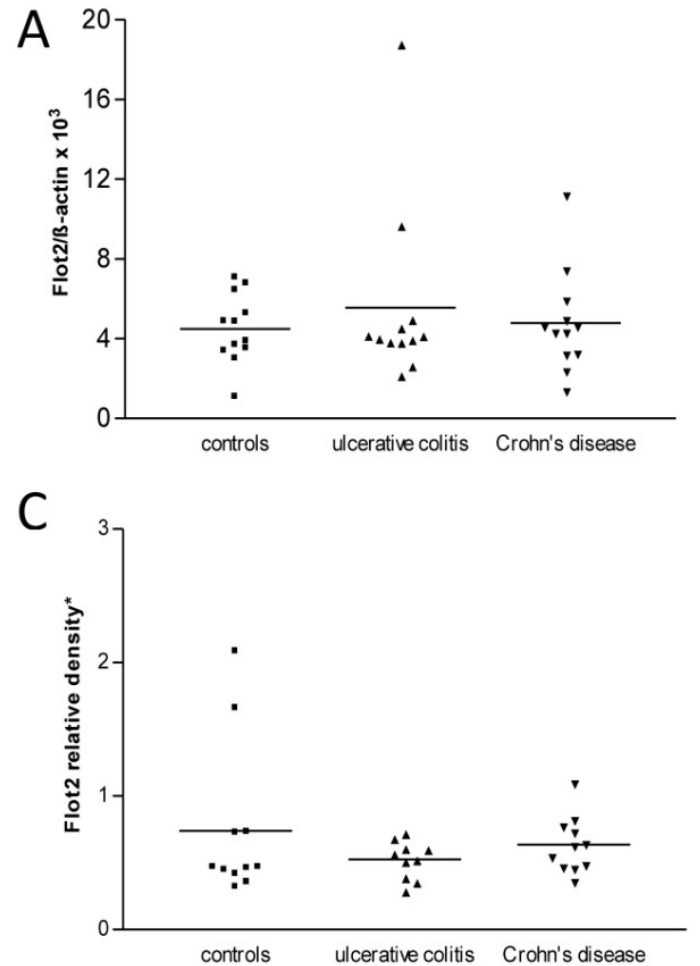

E

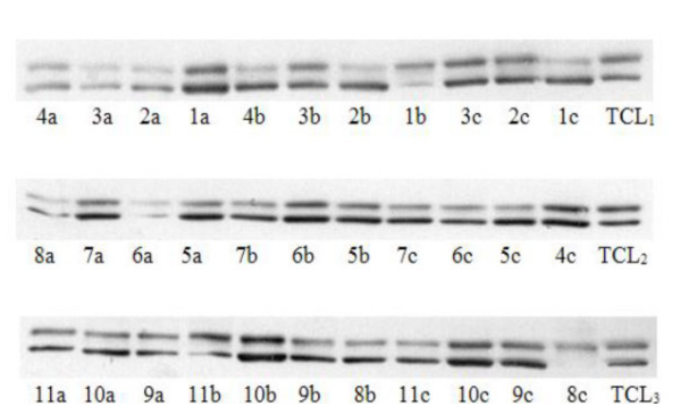

ated for their flotillin-2 expression by RT- PCR. In all, flotillin-2 expression was determined in 72 biopsies ( 3 $x 12$ from the ileum and the colon, 24 from controls, 24 from UC patients and 24 from CD patients). As shown in Figures 7A and B, flotillin-2 RNA expression levels did not show significant inter-group differences in either ileal or colonic biopsies (overall $p=0.98$ for ileal biopsies, overall $p=0.43$ for colonic biopsies). Comparative analyses of flotillin-2 RNA expression levels between ileal and colonic specimens revealed significantly higher expression in the colon than in the ileum, which proved true in all 3 patient groups $(\mathrm{p}<$ 0.0001 for controls, $p=0.009$ for UC patients, $p=0.02$ for $\mathrm{CD}$ patients).
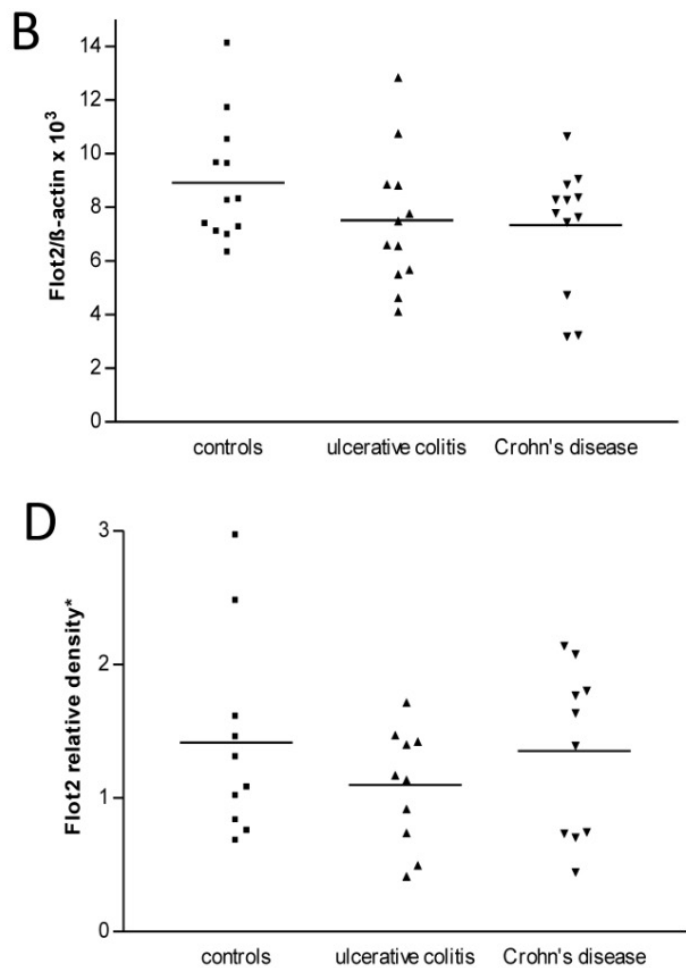

$\mathrm{F}$

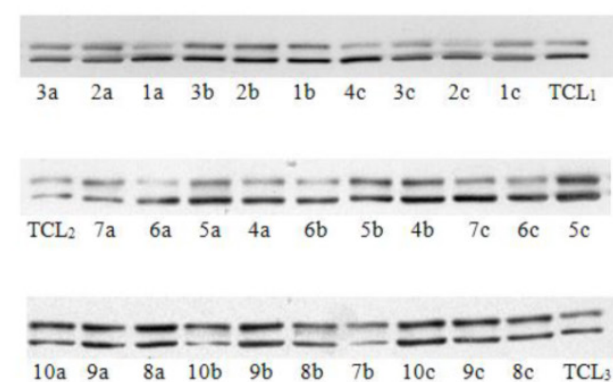

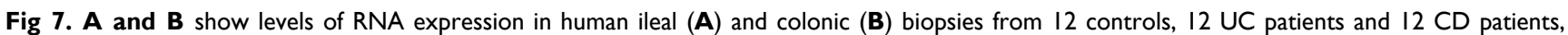

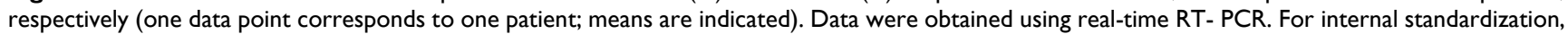
flotillin-2 values were related to human B-actin. Figure 7C displays results of flotillin-2 expression at the protein level, as achieved by Western blot analysis

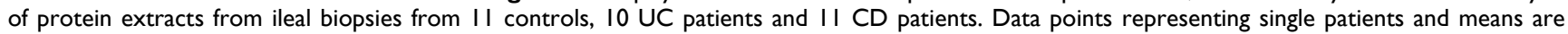

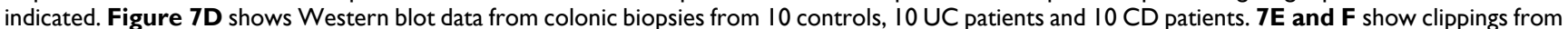

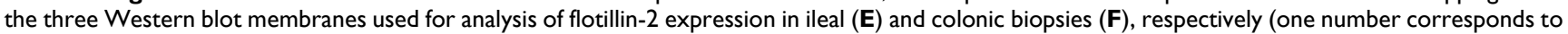
one biopsy; a: Crohn's disease, b: control, c: ulcerative colitis; TLC: total cell lysate). The upper bands represent flotillin-2, the lower bands represent B-actin. 


\section{Expression of flotillin-2 at the protein level - comparison between IBD and controls}

At the translational level, real-time RT-PCR data were confirmed by immunoblot analysis. Sixty-two biopsies from the ileum and colon were analyzed in this way. Thirty biopsies originated from the colon (10 controls, 10 UC patients, 10 CD patients), and 32 biopsies from the ileum (11 controls, 10 UC patients, 11 CD patients) (Figures 7E and F). Regarding the ileal biopsies, relative densitometric values of flotillin-2 bands referenced to $B$-actin bands and total cell lysate bands were $0.77 \pm 0.19$ in controls, $0.53 \pm 0.04$ in UC patients and $0.62 \pm 0.07$ in $\mathrm{CD}$ patients (overall $\mathrm{p}=$ 0.45 ) (Figure 7C). In the colonic biopsies, flotillin-2 expression likewise tended to be strongest in controls $(1.42 \pm 0.24)$ and slightly lower in patients with UC $(1.10 \pm 0.14)$ or CD $(1.35 \pm 0.20)$, without statistically significant intergroup differences (overall $p=0.62$ ) (Figure 7D). However, underlining the results obtained by RT-PCR, Western blot analysis yielded significantly higher flotillin-2 expression in colonic biopsies when compared to biopsies acquired from the ileum ( $p=0.01$ for controls, $p=0.005$ for UC patients, $p=0.02$ for CD patients) (Figure 7). It was also of interest to correlate disease activity of IBD patients with flotillin-2 expression in their biopsies. Table 1 shows, though, that the vast majority of patients with UC and $\mathrm{CD}$ suffered from active disease when the specimens were obtained. Thus subgroups were too small for reasonable subgroup analysis. It is important to note, though, that the difference between flotillin-2 expression in the ileum as compared to that in the colon was smaller for CD patients than for UC patients. This might be due to the fact that most UC patients had active inflammation in the colon, but not in the terminal ileum. In contrast, $78 \%(7 / 9)$ of the patients with active $C D$ at sample acquisition had not only colitis, but also ileitis (see Table 1).

\section{Discussion}

This study was designed to investigate whether flotillin-2 might be involved in the pathogenesis of IBD, especially in that of UC. The hypothesis of a disease-prompting or disease-modifying role of potential alterations to the expression of flotillin- 2 in the gut accrued from unpublished microarray data. These had revealed a reduced flotillin-2 RNA expression in biopsies from 4 UC patients when compared to controls.

In light of the data published by Kim et al., which showed an increased expression of flotillin-1 in autoimmune encephalitis, ${ }^{[19]}$ it seems at a first pass implausible to expect that the opposite, namely a reduced expression, of the related protein flotillin-2 may be a disease factor in UC. After all, UC has been considered to be a kind of autoimmune disease by some authors. However, taking into account the suggested functions of flotillin-2 in cells, such as regeneration, endocytosis and cell adhesion, any impairment of these by reduced flotillin-2 expression is indeed a factor to be considered as a potential initiator of inflammation, be it in the gut or elsewhere. Taking all of these facts together, it appeared intriguing to go further into this subject.

In order to test the hypothesis of a reduced flotillin-2 expression in the intestines of patients with $\mathrm{UC}$, experiments were first conducted in vitro and then in vivo based on those findings. It was of foremost interest to ascertain whether the potential changes in flotillin-2 expression in UC and maybe also in $\mathrm{CD}$ could be reproduced at the protein level.

As we were interested in a potential role for flotillin-2 in IBD, we chose to use CaCo- $2 \mathrm{w}$ cells as in vitro model first. The phenotype of $\mathrm{CaCo}-2 \mathrm{w}$ cells albeit derived from human colon carcinoma - resembles more that of small intestinal cells than that of colon cells when grown on filters to attain polarization. ${ }^{[37]}$ We considered CaCo- $2 \mathrm{w}$ cells, in both polarized and unpolarized states, to be an adequate model for our incipient in vitro experiments.

It is known that the expression of flotillins varies in different cell types and tissues. ${ }^{[13,14]}$ As in many other cell types in the human body, flotillin-2 was clearly expressed in CaCo-2w cells, being model cells of human enterocytes. There, flotillin- 2 was distinctly found in association with the plasma membrane, but also to a high degree in association with intracellular vesicles. Interestingly, we observed a shift in flotillin-2 expression to areas of cell-cell contact and, in parallel, a reduction of flotillin-2 expression in intracellular vesicles. This is in agreement with previously reported findings by Stuermer et al. and Langhorst et al. $[9,38,39]$ and underlines the suggested role of CaCo-2w cells in cell adhesion.[5]

Immunofluorescence microscopy revealed co-localization of flotlillin- 1 and flotillin- 2 in CaCo- $2 \mathrm{w}$ cells, supporting the notion that the two proteins can form hetero-oligomers. Accordingly, they have been observed together in other cells and tissues, ${ }^{66,40]}$ supporting the idea that this mutual interaction also occurs in the gut. In undifferentiated $\mathrm{CaCo}-2 \mathrm{w}$ cells we found vesicles containing flotillin-1 (and 2) to be either diffusely scattered or concentrated in the perinuclear region. After polarization, however, expression of flotillins proved to be strictly confined to a subapical compartment. A similar phenomenon has been observed in T cells, in which both flotillins were found localized in "preformed caps". Preformed caps are considered to be stable platforms with essential 
regulatory functions in $\mathrm{T}$ cell activation. ${ }^{[16,41]}$ These regulatory functions seem to mainly concern signal transduction and cytoskeleton dynamics. Therefore it may be concluded that flotillins also have an essential role in the differentiation of enterocytes, which are known for their high rate of cell turnover.

A novel and interesting finding of this study is that flotillin-1 (and -2), when looking only at the plasma membrane, seem to be confined to the basolateral compartment in polarized $\mathrm{CaCo}-2 \mathrm{w}$ cells, without appearance in the apical portion. Regarding this observation, we found no comparable data in the literature. The finding of a basolateral membrane compartmentalization of flotillins in enterocytes was confirmed in cryosections from human ileal and colonic biopsies. The absence of flotillins in the apical plasma membranes of enterocytes is striking, since flotillins are widely used as lipid raft markers, ${ }^{[4]}$ and lipid rafts are considered to be especially abundant in the apical cell membranes of enterocytes.[24, 25] Langhorst et al. have questioned the adequacy of flotillin-1 as a raft marker due to its prevailing intracellular localization. ${ }^{[6]}$ Based on the present data, it has to be considered once more whether flotillins can be employed as lipid raft markers in any cell line or tissue.

What does the basolateral localization of flotillins in enterocytes imply? The apical and basolateral poles of an epithelial cell have to meet rather different requirements. Major tasks of the basolateral cell membrane are reception and conversion of signals for proliferation, differentiation, migration and apoptosis. The apical pole, in contrast, mainly serves in absorption of nutrients and protection against aggressive external factors like acid, digestive enzymes and pathogens. We speculate that the basolateral localization of flotillins in enterocytes is associated with signal transduction and cytoskeletal dynamics, while the subapical, flotillin-containing vesicles are involved in endocytosis.

Knowing that flotillin-2, in line with the above-mentioned microarray data, is expressed in human enterocytes of the ileum and colon, we proceeded to a simple cell model of colitis to screen whether flotillin-2 expression was strongly influenced by pro-inflammatory signals. It was shown previously in the same laboratory that TNFa, added to the medium of sub-confluent $\mathrm{CaCo}-2 \mathrm{w}$ cells, leads to a time-dependent, efficient nuclear translocation of the p65 subunit of NF-KB as soon as 15 min after addition of TNFa. ${ }^{[33]} \mathrm{TNFa}$ levels have been demonstrated to be increased in the intestinal mucosa from patients with $\mathrm{CD}$ and $\mathrm{UC}$, and mucosal cultures from patients with the two diseases proved to produce more $\mathrm{TNFa}$, IL-1 $\beta$, and IL-6 compared to normal mucosa. ${ }^{[42]}$ In addition, anti-TNFa antibodies are used successfully in the treatment of IBD. Furthermore, lipid rafts, with which flotillins are considered to be associated, play a role in the activation of the NFKB cascade via TNFa activation.[26] However, immunostaining of TNFa-stimulated CaCo- $2 \mathrm{w}$ cells as compared to untreated cells did not show obvious differences in intracellular flotillin-2 localization. Of course these are no quantitative data. This experiment was interesting for another reason: Numerous phenomena have been published as potential actors in the pathogenesis of IBD. Many of them could not be clearly determined to be primary pathogenetic factors. For them, the chicken-or-egg question remains largely unanswered: does an alteration precede and thus cause inflammation, or does inflammation cause the alteration?

The TNFa data mentioned above could suggest that the exact sampling site of biopsies in the inflamed colon in the present study is not of great importance. Still, we sought to avoid biopsy collection from severely inflamed tissue, even though areas with slight or only microscopically visible inflammation were included.

In contrast to the above-mentioned microarray data showing a 5-fold reduction in flotillin-2 expression in UC patients, flotillin-2 expression in the human intestine had indeed a tendency to be lower in UC patients compared to healthy subjects, but no significant inter-group difference was established. This was not only the case at the RNA level but, more importantly, likewise at the protein level, as shown by immunoblotting. Since sample numbers were higher in this study and as expression analyses were also performed at the protein level, we believe that the present results outweigh the previous microarray data. Thus, our data do not imply a major role for flotillin-2 in the pathogenesis of IBD, even though they do not exclude a role of flotillin-2 in IBD for certain. A limiting factor is that sample numbers were small and the distribution of disease activities in the IBD groups was inhomogeneous. In view of the role of flotillin-2 in regenerative processes it might be worthwhile investigating its expression during the recovery phase induced by medication in order to exclude a role of flotillin-2 in IBD for certain. IBD patients included in our study suffered from UC or CD in equal numbers. The specimens represented a variety of different disease activities. The fact that macroscopically unaffected mucosal areas were preferred for sampling does not rule out the possibility that the measured flotillin-2 expression might have been influenced by inflammation in some cases. In future experiments, it might be interesting to take biopsies from inflamed and non-inflamed areas in the same patient. 
The stronger expression of flotillin-2 in the colon compared to the ileum, even in healthy subjects, is remarkable and raises the question as to whether flotillin-2 possesses a special function in the colon. Major functions of the colon are absorption of water and electrolytes, while the ileum is mainly responsible for the absorption of vitamin B12, bile acids and remaining products of digestion entering from the jejunum. However, the colon and the ileum, the latter being mechanically separated from the cecum via the ileocecal valve, also differ greatly in the amounts of bacteria populating their lumina. The colon contains ca. $10^{11}$ to $10^{12} \mathrm{CFU} / \mathrm{mL}$ stool, whereas the ileum has bacterial concentrations of about $10^{4}$ to $10^{7} \mathrm{CFU} / \mathrm{mL}$ stool.[43] Yet our data are not sufficient to imply a role of flotillin-2 in defense of the host against intestinal bacteria. However, this could be an interesting starting point for further research, notably as we found flotillin-2 within the basolateral cell membranes of enterocytes, which are not in direct contact with bacteria.

\section{Conclusions}

Flotillin-2 is distinctly expressed in human enterocytes, where it concentrates at the basolateral plasma membrane and in subapical compartments, but interestingly not at the apical plasma membrane. This might imply that in the intestine its main function relates to cytoskeleton dynamics and signal transduction, as well as endocytosis. Its higher expression in the colon as compared to the ileum may indicate that flotillin-2 has an especially important role in the colon.

Our hypothesis of a reduced flotillin-2 expression in IBD - mainly ulcerative colitis - versus controls could not be confirmed by the present data, which were acquired at the level of RNA as well as protein. Thus, these findings do not suggest a major role for flotillin-2 specific to the pathogenesis of IBD, even though the sample numbers were small.

\section{Abbreviations}

BCA bicinchoninic acid, CD Crohn's disease, CEA carcinoembryonic antigen, CFU colony forming unit, ESCC esophageal squamous cell carcinoma, IBD inflammatory bowel disease, PCR polymerase chain reaction, PFA paraformaldehyde, RT reverse transcriptase, UC ulcerative colitis.

\section{Acknowledgments}

We thank Sabine Tuma, Karin Bents and Richard Sparla for expert technical support. This publication contains parts of the medical thesis of Inga Buchholz. We thank Kai Simons (MPI Dresden, Germany) for providing flotillin-1 antibodies and Jerrold R. Turner
(University of Chicago, IL) for providing Caco-2w cells.

\section{Competing Interests}

The authors have declared that no competing interest exists.

\section{References}

1. Morrow IC, Parton RG. Flotillins and the PHB domain protein family: rafts, worms and anaesthetics. Traffic 2005; 6:725-740.

2. Schulte T, Paschke KA, Laessing U, et al. Reggie-1 and reggie-2, two cell surface proteins expressed by retinal ganglion cells during axon regeneration. Development 1997; 124: 577-587.

3. Lang DM, Lommel S, Jung $\mathrm{M}$, et al. Identification of reggie-1 and reggie-2 as plasmamembrane-associated proteins which cocluster with activated GPI-anchored cell adhesion molecules in non-caveolar micropatches in neurons. J Neurobiol 1998; 37: 502-523.

4. Browman DT, Hoegg MB, Robbins SM. The SPFH domain-containing proteins: more than lipid raft markers. Trends in Cell Biology 2007; 17: 394-402.

5. Banning A, Tomasovic A, Tikkanen R. Functional aspects of membrane association of flotillins. Curr Protein Pept Sci 2011; 12: 725-735.

6. Langhorst MF, Reuter A, Stuermer CAO. Scaffolding microdomains and beyond: the function of reggie/flotillin proteins. Cell Mol Life Sci 2005; 62: 2228-2240.

7. Solomon S, Masilamani M, Rajendran L, et al. The lipid raft microdomain-associated protein reggie-1/flotillin-2 is expressed in human B cells and localized at the plasma membrane and centrosome in PBMCs. Immunobiology 2002; 205: 108-119.

8. Gagescu R, Demaurex N, Parton RG, et al. The recycling endosome of Madin-Darby canine kidney cells is a mildly acidic compartment rich in raft components. Mol Biol Cell 2000; 11: 2775-2791.

9. Stuermer C A, Langhorst MF, Wiechers MF, et al. PrPc capping in T cells promotes its association with the lipid raft proteins reggie-1 and reggie-2 and leads to signal transduction. FASEB 2004; 18: 1731-1733.

10. Dermine JF, Duclos S, Garin J, et al. Flotillin-1-enriched lipid raft domains accumulate on maturing phagosomes. J Biol Chem 2001; 276: 18507-18512.

11. de Gassart A, Geminard C, Fevrier B, et al. Lipid raft-associated protein sorting in exosomes. Blood 2003; 102: 4336-4344.

12. Langui D, Girardot N, El Hachimi KH, et al. Subcellular topography of neuronal Abeta peptide in APPxPS1 transgenic mice. Am J Pathol 2004; 165: 1465-1477.

13. Otto GP, Nichols BJ. The roles of flotillin microdomains - endocytosis and beyond. J Cell Science 2011; 124: 3933-3940.

14. Volonté D, Galbiati F, Li S, et al. Flotillins/Cavatellins are differentially expressed in cells and tissues and form a hetero-oligomeric complex with caveolins in vivo. Characterization and epitope-mapping of a novel flotillin-1 monoclonal antibody probe. J Biol Chem 1999; 274: 12702-12709.

15. Edgar AJ, Polak JM. Flotillin-1: gene structure: cDNA cloning from human lung and the identification of alternative polyadenylation signals. Int J Biochem Cell Biol 2001; 33: 53-64.

16. Rajendran L, Masilamani M, Salomon S, et al. Asymmetric localization of flotillins (reggies) in preassembled platforms confers inherent polarity to hematopoietic cells. PNAS 2003; 100: 8241-8246.

17. Zhao F, Zhang J, Liu Y S, et al. Research advances on flotillins. Virol J 2011; 8: 479

18. Lin C, Wu Z, Lin X, et al. Knockdown of FLOT1 impairs cell proliferation and tumorigenicity in breast cancer through upregulation of FOXO3a. Clin Cancer Res 2011; 17: 3089-3099.

19. Kim H, Ahn M, Moon C, et al. Immunohistochemical study of flotillin-1 in the spinal cord of Lewis rats with experimental autoimmune encephalitis. Brain Res 2006; 1114: 204-211.

20. Song L, Gong H, Lin C, et al. Flotillin-1 promotes tumor necrosis factor-a signaling and activation of NF- $\mathrm{kB}$ in esophageal squamous cell carcinoma cells. Gastroenterology 2012; 143: 995-1005.

21. Strober W, Fuss I, Mannon P. The fundamental basis of inflammatory bowel disease. J Clin Invest 2007; 117: 514-521.

22. Bowie RV, Donatello S, Leyes C, et al. Lipid rafts are disrupted in mildly inflamed intestinal microenvironments without overt disruption of the epithelial barrier. Am J Physiol Gastrointest Liver 2012; 302: G781-793.

23. Treede I, Braun A, Jeliaskova P, et al. TNF-alpha-induced up-regulation of pro-inflammatory cytokines is reduced by phosphatidylcholine in intestinal epithelial cells. BMC Gastroenterol 2009 Jul 13;9:53. 
24. Danielsen EM, Hansen GH. Lipid raft organization and function in brush borders of epithelial cells. Mol Membr Biol 2006; 23: 71-79.

25. Nguyen HT, Amine AB, Lafitte D, et al. Proteomic characterization of lipid rafts markers from the rat intestinal brush border. Biochem Biophys Res Commun 2006; 342: 236-244.

26. Legler DF, Micheau O, Doucey MA, et al. Recruitment of TNF receptor 1 to lipid rafts is essential for TNFalpha-mediated NF-kappaB activation. Immunity 2003; 18: 655-64.

27. Schmitz G, Orso E. CD14 signalling in lipid rafts: new ligands and co-receptors. Curr Opin Lipidol 2002; 13: 513-521.

28. Triantafilou MS, Morath S, Mackie A, et al. Lateral diffusion of Toll-like receptors reveals that they are transiently confined within lipid rafts on the plasma membrane. J Cell Sci 2004; 117: 4007-4014.

29. Kalischuk LD, Inglis GD, Buret AG. Campylobacter jejuni induces transcellular translocation of commensal bacteria via lipid rafts. Gut Pathog 2009 Feb 3;1(1):2.

30. Lafont F, van der Goot FG. Bacterial invasion via lipid rafts. Cell Microbiol 2005; 7: 613-620.

31. Stremmel W, Merle U, Zahn A, et al. Retarded release phosphatidylcholine benefits patients with chronic active ulcerative colitis. Gut 2005; 54: 966-71.

32. Stremmel W, Ehehalt R, Autschbach F, Karner M. Phosphatidylcholine for steroid-refractory chronic ulcerative colitis: a randomized trial. Ann Intern Med 2007; 147: 603-610.

33. Treede I, Braun A, Sparla R, et al. Anti-inflammatory effects of phosphatidylcholine. J Biol Chem 2007; 282: 27155-27164.

34. Moehle C, Ackermann N, Langmann T, et al. Aberrant intestinal expression and allelic variants of mucin genes associated with inflammatory bowel disease. J Mol Med 2006; 84: 1055-1066.

35. Zahn A, Moehle C, Langmann T, et al. Aquaporin-8 expression is reduced in ileum and induced in colon of patients with ulcerative colitis. World J Gastroenterol 2007; 13: 1687-1695.

36. Stoscheck CM. Quantitation of protein. Methods Enzymol 1990; 182: $50-68$.

37. Pinto M, Robine-Léon S, Appay MD, et al. Enterocyte-like differentiation and polarization of the human colon carcinoma cell line Caco-2 in culture. Biol Cell 1983; 47: 323-330.

38. Stuermer CA, Lang DM, Kirsch F, et al. Glycosylphosphatidyl inositol-anchored proteins and fyn kinase assemble in noncaveolar plasma membrane micro-domains defined by reggie- 1 and -2 . Mol Biol Cell 2001; 12: 3031-3045.

39. Langhorst MF, Reuter A, Jaeger FA, et al. Trafficking of the microdomain scaffolding protein reggie-1/flotillin-2. Eur J Cell Biol 2008; 87: 211-226.

40. Solis GP, Hoegg M, Munderloh C, et al. Reggie/flotillin proteins are organized into stable tetramers in membrane microdomains. Biochem J 2007; 403: 313-322.

41. Slaughter N, Laux I, Tu X, et al. The flotillins are integral membrane proteins in lipid rafts that contain TCR-associated signaling components: implications for T-cell activation. Clin Immunol 2003; 108: 138-151.

42. Reimund JM, Wittersheim C, Dumont S, et al. Increased production of tumor necrosis factor-alpha interleukin-1 beta, and interleukin- 6 by morphologically normal intestinal biopsies from patients with Crohn's disease. Gut 1996; 39: 684-689.

43. Sears CL. A dynamic partnership: celebrating our gut flora. Anaerobe 2005; 11: 247-251. 